\title{
Design of erbium doped silicon nanocavities for single photon applications
}

\author{
Matteo Di Giancamillo ${ }^{1,2}$, Paolo Biagioni ${ }^{2}$, Vito Sorianello ${ }^{3}$ and Enrico Prati $^{1 *}$ \\ ${ }^{1}$ Istituto di Fotonica e Nanotecnologie, Consiglio Nazionale delle Ricerche, 20133 Milano, Italy \\ ${ }^{2}$ Dipartimento di Fisica, Politecnico di Milano, 20133 Milano, Italy \\ ${ }^{3}$ Photonic Networks and Technologies Lab, Consorzio Nazionale Interuniversitario per le Telecomunicazioni (CNIT), 56124 Pisa, Italy
}

\begin{abstract}
Silicon-based quantum communication technologies are becoming a factual reality. However, the challenges related to an earth-space unifying technology are several, and nowadays an integrated source compatible with the CMOS technology is still missing. Here we present the design of a weak photon source consisting of a LED able to emit directly into the optical circuit and obtained through the doping of a portion of a silicon waveguide with $\mathrm{ErO}_{\mathrm{x}}$ complexes. To enhance the radiative emission, the source is placed inside a resonant cavity delimited by two waveguide Bragg mirrors. A study on the performance of the device is carried out as a function of different parameters, such as the geometry of the cavity and of the contacts used to electrically excite the defects, the doping level, and the characteristics of the mirrors. We design a prototype that guarantees a Purcell factor in the order of tens, emitting ideally $10^{7}-10^{8}$ photons per second. The simulations provide a promising ground to further develop fully integrated single photon sources in silicon photonic circuits.
\end{abstract}

\section{Introduction}

The goal of the research is to design a LED weak photon source for silicon-based quantum communications in space at a wavelength of $1540 \mathrm{~nm}$, through the radiative de-excitation of $\mathrm{ErO}_{\mathrm{x}}$ complexes generated by erbium implant in a portion of a silicon waveguide, and to evaluate the total number of photons emitted per second as a function of different parameters. The aim of the source is to generate photons directly in the optical guide and to use materials compatible with the CMOS technology, making it competitive in terms of costs and performance. Nowadays, a commercial device that satisfies all the demands for a ground-space technology and where the native generation and detection of single photons and their polarization states is integrated on the same chip does not exist $[1,2]$, even if some of the Authors already explored nano-LEDs based on Er [3]. The use of silicon has the potential to combine the advantages and knowledge of electronic control based on CMOS microelectronic technology with those deriving from the integration of silicon photonics, increasing the tolerance to temperature variations that could undermine the performance of non-monolithic architectures [1,2].

Fig. 1 represents a top-view sketch of the proposed device. A potential difference is applied to two contacts made of doped $\mathrm{Si}$, which are placed at the sides of the waveguide and excite the erbium atoms through carrier-mediated excitation [4]. To enhance the radiative emission, the doped region, which acts as the source, is placed inside a cavity tuned to be resonant with the emitted wavelength and obtained through two waveguide Bragg mirrors [5]. The waveguide core, with a width of $480 \mathrm{~nm}$ and a height of $220 \mathrm{~nm}$, is realized on top of the silicon oxide layer of a SOI wafer and surrounded by a thin layer of $100 \mathrm{~nm}$ thickness of the same oxide material.

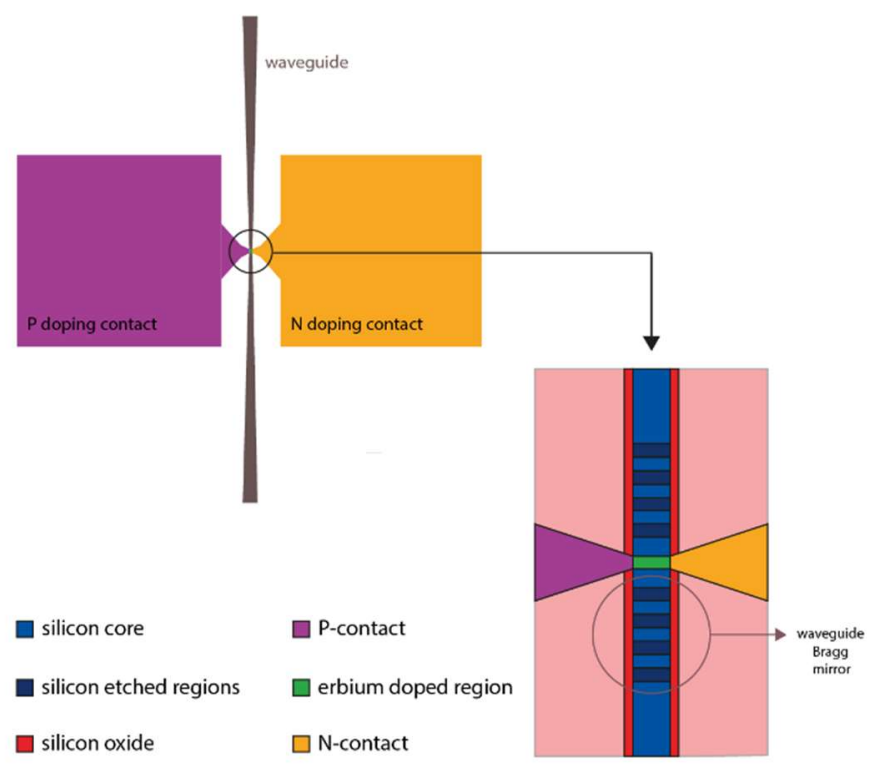

Fig. 1. Sketch of the device.

The present work focuses on the electromagnetic design of the waveguide and of the optical cavity, in order to

\footnotetext{
$\overline{\text { * Corresponding author: enrico.prati@enr.it }}$
} 
optimize their geometry. It is carried out using two simulation software packages, Ansys Lumerical [6] and Comsol Multiphysics [7], taking all the technical requirements and the manufacturing constraints into account. To model the erbium-doped silicon material, we related the concentration of dopants to the variation of the real and imaginary part of the refractive index of the host material following Ref. 8.

\section{Results and discussion}

The results of the simulations are divided into two sections: the first one is focused on the propagation within the cavity, while the second one concerns the characteristics and properties of the cavity resonance.

Propagation The following results are obtained with the Comsol Multi-physics wave optics module. An automatic mesh controlled by the software and based on the physics of the problem has been selected. A mode analysis of the cavity is performed to evaluate the effective mode index of the cavity, which results to be $n_{1}=2.402$ for the fundamental mode and $\mathrm{n}_{2}=1.681$ for the second mode, both represented in Fig. 2. By exploiting a vectorial representation of the electric field, one can appreciate that the fundamental mode of the waveguide possesses a dominant TE character and is polarized perpendicular to the second mode, which is mainly TM. There are no other propagating modes. As we deal with resonant effects in the waveguide, one should also notice that the resonant mode in the cavity only involves the TE mode, while the $\mathrm{TM}$ is suppressed along propagation in the optical circuit.

a

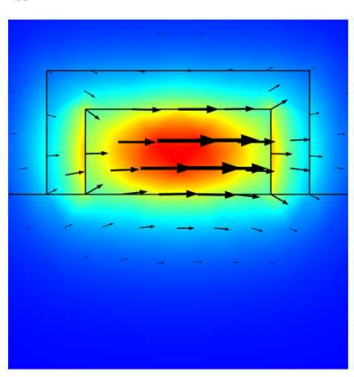

b

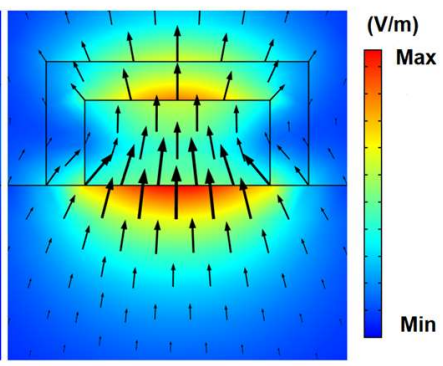

Fig. 2. Direction of the electric field. In all the points simulated the electric field vector is represented by an arrow whose components are proportional to the in-plane and out-of-plane field components. (a) Fundamental mode (TE), (b) second mode (TM).

The effective mode index is calculated by considering an erbium doping concentration of $10^{17} \mathrm{~cm}^{-3}, 10^{18} \mathrm{~cm}^{-3}$ and $10^{19} \mathrm{~cm}^{-3}$ (Table 1). While the real part of the mode index is negligibly affected by the doping, the imaginary part increases up to $10^{-2}$ for the highest concentration, still it does not represent the main source of losses in the cavity, whose performance is dominated by mirror losses and scattering losses, as discussed later on. It is also important to notice that the real part of the refractive index of the erbium doped area is very close to the undoped one. This is a fundamental prerequisite, since it avoids significant mismatch between mode indices inside and outside of the doper area. A larger mismatch would cause scattering and reflection of the light. We can conclude, from this analysis, that the doping level is irrelevant for this major aspect, and the optical properties of the material are not sensibly modified, especially considering that the maximum investigated doping value of $10^{19} \mathrm{~cm}^{-3}$ is difficult to obtain using standard techniques.

\begin{tabular}{|l|l|l|l|}
\hline Doping concentration & $10^{17} \mathrm{~cm}^{-3}$ & $10^{18} \mathrm{~cm}^{-3}$ & $10^{19} \mathrm{~cm}^{-3}$ \\
\hline Effective mode index & $2.4133-8.2 \mathrm{E}-5 \mathrm{i}$ & $2.4123-0.0012 \mathrm{i}$ & $2.4022-0.0180 \mathrm{i}$ \\
\hline
\end{tabular}

Table 1. Effective mode index for three different erbium doping levels of the silicon waveguide.

The mode effective index of the etched waveguide $(70 \mathrm{~nm}$ etching depth) for the realization of the Bragg mirror is $\mathrm{n}_{\text {etch }}=2.060$. Such a value is exploited to design the periodicity of the fibre Bragg mirror. Considering now the full 3D structure, an analysis on the losses of the cavity, due to the presence of the dopants and the contacts, was performed. In Fig. 3 the transmission along the doped region of the waveguide is plotted as a function of the length of the doped area (and therefore of the lateral contacts), for a cavity with and without contacts. The graph shows that the doping level, which is fixed at $10^{18} \mathrm{~cm}^{-3}$, does not significantly influence the overall losses of the cavity, which are instead determined mainly by the presence of the contacts.

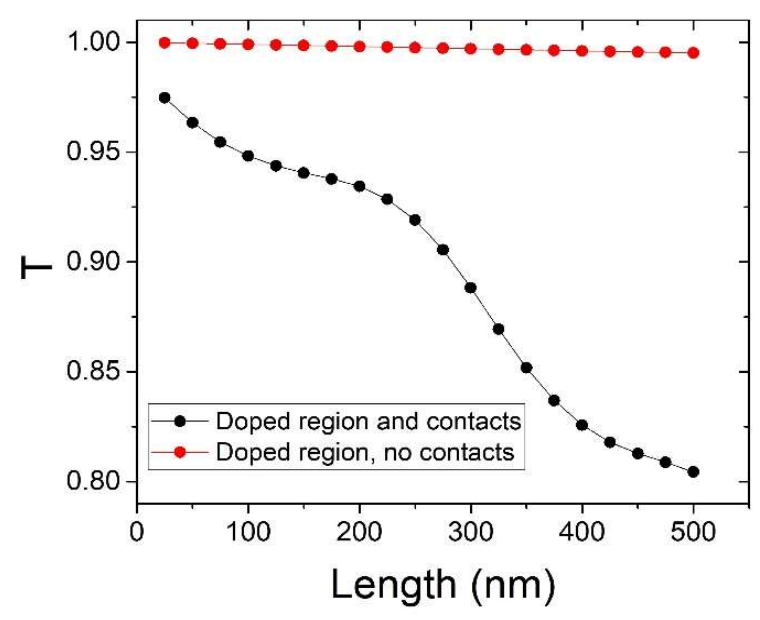

Fig. 3. Transmission coefficients from the input to the output port of the cavity as a function of the length of the doped area and therefore of the lateral contacts.

Cavity analysis The cavity is designed considering two waveguide Bragg mirrors as reported in Fig. 4, which are characterized by a periodic variation in the mode index of the waveguide core obtained by etching the height of the core by $70 \mathrm{~nm}$. According to the mentioned effective mode indices and the etching depth, the grating periodicity for a stopband mirror centred at $1540 \mathrm{~nm}$ is around $344 \mathrm{~nm}$. The reflection and transmission coefficients of the mirror, which is reported in Fig. 5 
together with the norm of the electric field along the longitudinal cut plane, starts to saturate with a number of periods roughly equal to 40 , with a value approximately equal to $96 \%$.

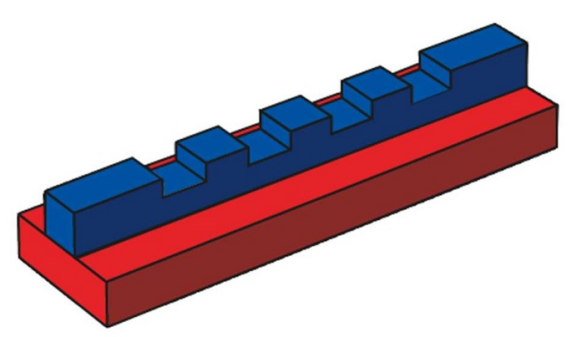

Silicon core

Buried oxide

Fig. 4. Waveguide Bragg mirror after the etching process. The silicon core is shaped to obtain a periodic discontinuity of the refractive index. In this conceptual view the upper cladding of $100 \mathrm{~nm}$ thickness is not drawn, but it is present in all the simulations.

The mirror effectively dampens the electric field and the reflectivity saturates after a specific length of the mirror because no electric field is present within the structure after a sufficiently large number of periods.

a

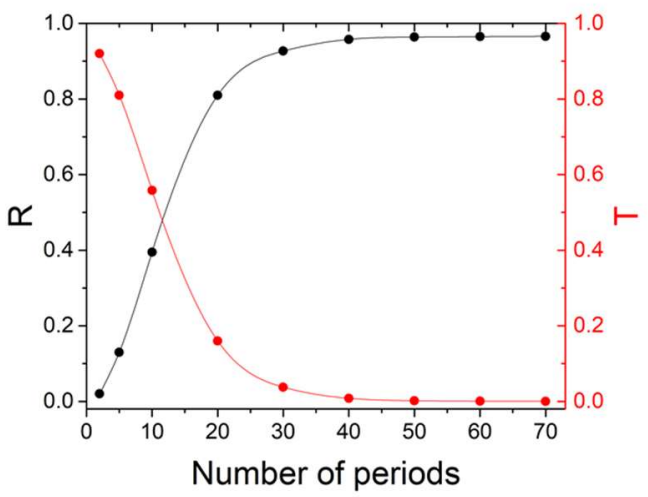

b

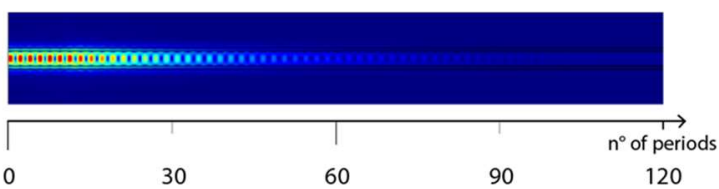

Fig. 5. (a) The trend of the reflection and transmission coefficients as a function of the number of periods, (b) the norm of the electric field along the longitudinal cut plane.

The sum between $\mathrm{R}$ and $\mathrm{T}$ is in the order of $97 \%$. This is because the core discontinuities, due to the etching, scatter part of the light towards the lower or upper cladding. Finally, the spectral response for a mirror with 35 periods is reported in Fig. 6. The spectrum is well centred and it has a reflectivity in the order of $90 \%$ between 1520 and $1560 \mathrm{~nm}$. This stopband, which is determined by the mismatch between the two effective mode indices and therefore by the etching depth, is matched to the width of the erbium emission peak at room temperature. It cannot be enlarged further since a deeper etching corresponds also to larger scattering losses.

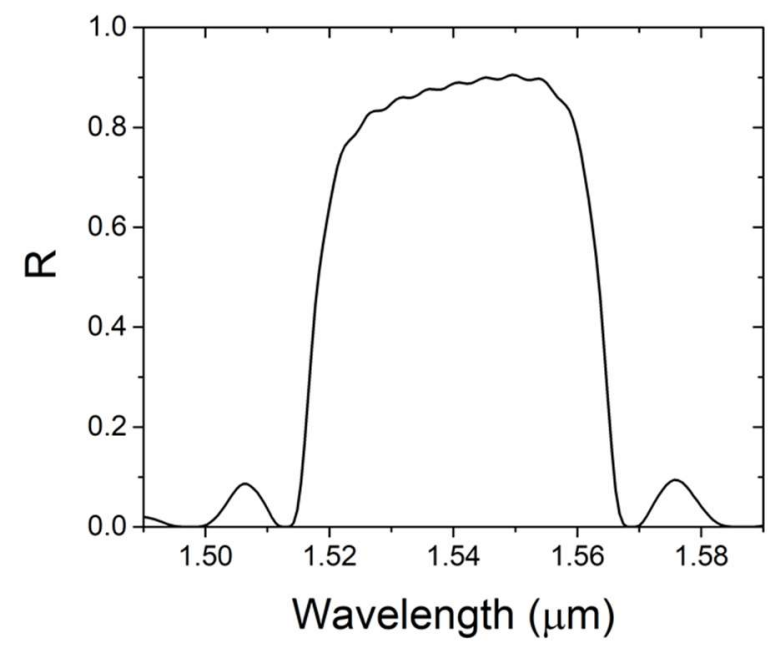

Fig. 6. Spectral response of the waveguide Bragg mirror with 35 periods, a periodicity of $344 \mathrm{~nm}$, and an etching depth of $70 \mathrm{~nm}$.

In order to evaluate the Purcell factor, which returns the enhancement of the spontaneous emission rate of a quantum system by its environment, an electric dipole source emitting at $1540 \mathrm{~nm}$ is placed in the cavity, coupled to the TE mode, and surrounded by a transmission box to evaluate the difference of the emission with respect to the free space, as shown in Fig. 7. A parametric study of the length of the cavity was carried out with Ansys Lumerical to obtain the lengths that maximize this effect, i.e. those resonant with the emission wavelength, which are $344 \mathrm{~nm}$ (half-wavelength cavity) and $644 \mathrm{~nm}$ (one-wavelength cavity).

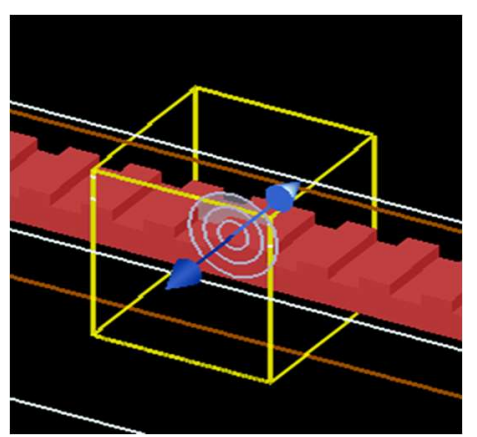

Fig. 7. Electric dipole source surrounded by the transmission box.

The results obtained with these simulations must be divided by two to consider the standing-wave modulation of the density of states along the cavity and then divided further by 3 to take into account all the possible spatial 
orientations of the dipole, ending up with a Purcell factor approximately equal to 10 .

a

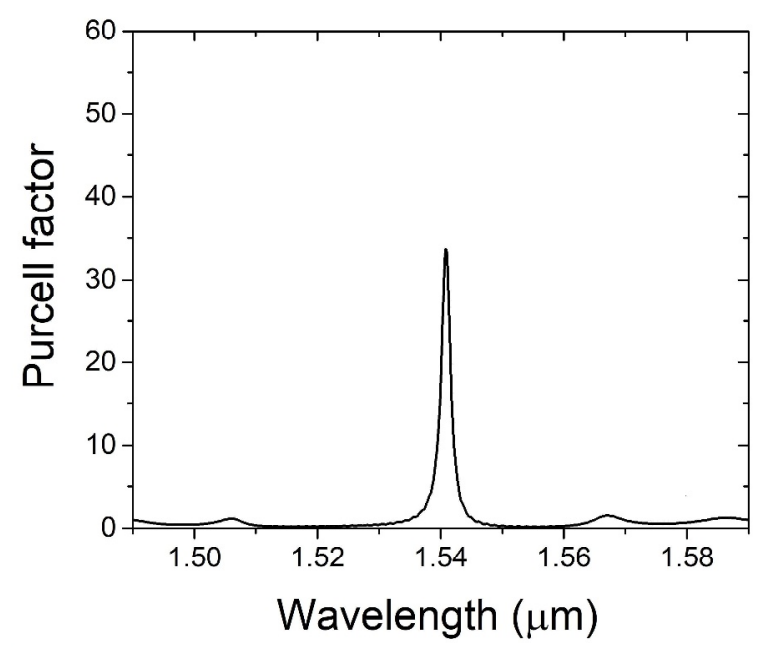

b

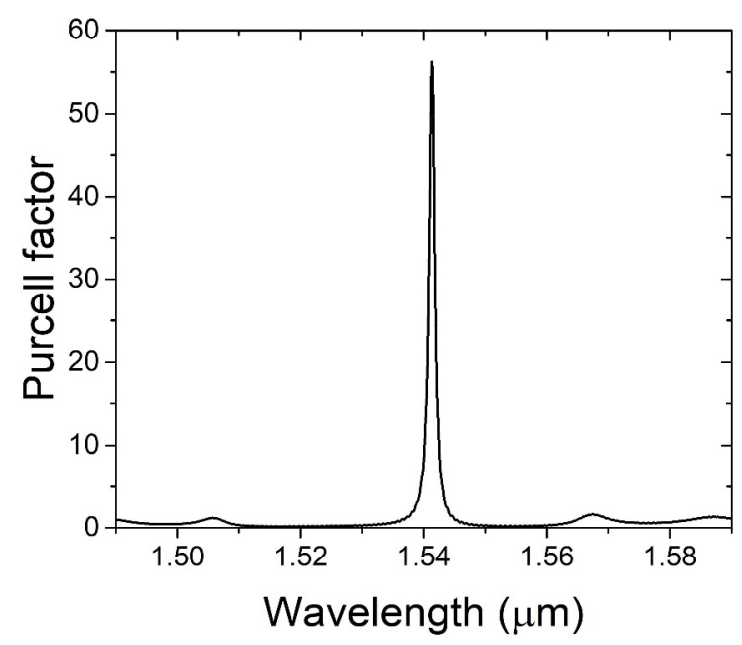

Fig. 8. Purcell factor of a broadband source mimicking $\mathrm{Er}$ emission for a cavity length of $344 \mathrm{~nm}$ (upper plot) and $644 \mathrm{~nm}$ (lower plot).

The difference between the two graphs could be due to different scattering losses when the mode meets the mirror. In fact, some of the scattered light can be 'recycled' into the cavity [9] and this phenomenon could be different for different cavity lengths. Finally, it is necessary to highlight that the simulations in Fig. 8 did not take doping into account, since the numerical code becomes unstable when the emitting dipole is immersed in an absorbing region.

\section{Conclusions}

This work provides a first systematic assessment of the perspective performance of a nanocavity LED emitter. Considering a volume of the doped region equal to $480 \mathrm{x}$ $220 \times 100 \mathrm{~nm}^{3}$ and a doping concentration of $10^{18} \mathrm{~cm}^{-3}$ we obtain a total number of emitting ions in the order of $10^{4}$. Since about one atom out of ten typically emits, considering the previously calculated Purcell factor and a radiative lifetime of $0.1-1 \mathrm{~ms}$, we expect a rate of emitted photons at least of the order of $10^{7}-10^{8} \mathrm{~s}^{-1}$ in continuouswave operation and even higher by pulsing the LED. This supports the feasibility of a device that meets the requirements of operation and compatibility with the manufacturing processes and growth of silicon in modern electronics.

\section{Acknowledgements}

This research was part of the QUASIX project funded by the Italian Space Agency (http://www.quasix.space/).

\section{References}

1. I. Aharonovich, D. Englund, M. Toth, Nat. Phot., 10, 631-641 (2016).

2. F. Cavaliere et al., Quantum Reports 2.1, 80-106 (2020)

3. T. Fujimoto et al., IEEE Silicon Nanoelectronics Workshop (SNW), 123-124 (2020)

4. A. Kenyon, Semicond. Sci. \& Tech., 20 (2005)

5. M. Pruessner, T. Stievater, W. Rabinovich, Opt. Lett., 32, 533-5 (2007)

6. Inc. C. COMSOL [Internet]. 2020. Available from: http://www.comsol.com/products/multiphysics/

7. Lumerical Inc. FDTD: 3D Electromagnetic Simulator

8. M. Nedeljkovic, R. Soref, G. Z. Mashanovich, IEEE Phot. J., 3, 1171-1180 (2011)

9. Ph. Lalanne, S. Mias, and J.P. Hugonin, Opt. Exp. 12, 458-467 (2004) 